\title{
Actitudes Culturales y Salud ${ }^{1}$
}

\begin{abstract}
Arturo G. Rillo
Departamento de Investigación Cientifica de la UAEM,

La salud está determinada por la organización social... y el Estado no debe permitir que el precio del neoliberalismo se pague con la salud del pueblo.
\end{abstract}

Federico Ortiz Quezada, 1986

\section{Introducción}

Tratar de reflexionar sobre las actitudes culturales y la salud en tiempo presente, es una ardua labor debido a las implicaciones que ambos elementos, tanto la cultura como la salud, tiene para el ser humano. Esta laboriosidad, a la que se agrega una extrema complejidad, se ve representada en la multiplicidad de trabajos que han integrado cada una de las mesas que se desarrollarán durante este seminario.

Ante esta evidencia, y considerando el interés de compartir una serie de reflexiones, la presente comunicación pretende revisar brevemente dos variables como lo son cultura y salud, dentro del contexto del paradigma de la transición, en particular la transición demográfica, la transición epidemiológica y la transición de la atención a la salud, que al interaccionar en el campo de lo social nos conduce a una respuesta social organizada a los problemas de salud. Para este propósito, el desarrollo de este trabajo contempla el desarrollo de los siguientes elementos:

a) El enfoque histórico del Proceso Salud-Enfermedad.

b) Una interpretación de la cultura desde la perspectiva del Proceso Salud-Enfermedad.

c) A partir de estos dos temas centrales, considerados como variables, se identifica la necesidad de analizar las relaciones que establecen la cultura y la salud, para propiciar la aparición de una respuesta social organizada que enfrente la problemática de salud que manifiesta la sociedad.

1 Conferencia Magistral presentada el 22 de Enero de 1996 en el "Seminario Interdisciplinario: Actitudes Culturales y Salud”, realizado del 22 al 26 de Enero de 1996 en la Cd. de Toluca, Estado de México; Universidad Autónoma del Estado de México. 
d) Este análisis, se inserta dentro del ámbito de lo que he llamado el "paradigma transicional" y que incluye la transición demográfica, la transición epidemiológica y la transición de la atención a la salud.

e) A partir, y si aceptamos el punto anterior, de los elementos proporcionados por el "paradigma transicional", podremos identificar diversas relaciones entre las actitudes culturales y la salud, entre las que destacan la inclusión social, la igualdad de oportunidades, la autonomía individual y familiar, la corresponsabilidad social, y el bienestar como la resultante final de la sumatoria entre cultura y salud.

f) Finalmente, se analizará si todos estos elementos contribuyen a obtener una respuesta social organizada para la salud que permita incrementar el estado de salud del individuo y de su comunidad.

\section{A) Enfoque histórico del Proceso Salud-Enfermedad}

La medicina, arte y ciencia que procura mitigar el sufrimiento humano, ha ido en desbandada en la búsqueda de la verdad científica, creando sistemas y teorías en la creencia de encontrar la ciencia reconfortadora, encontrándose el médico en el seno de una intensa lucha por la búsqueda de esta inquietante verdad, sin ver en la salud más que enfermedad, pasando desapercibido su aspecto fantasmagórico, contradictorio y limitante, que termina por derrumbar al individuo mismo.

El modo como se relaciona el hombre con su medio ambiente y con su sociedad, ha determinado el desarrollo de la medicina, desarrollo que ha estado sujeto al concepto prevalente de salud y enfermedad, conceptos que a través de la historia han recibido diversas acepciones, haciendo manifiesto el proceso histórico en el que las ciencias médicas se han desenvuelto.

En las culturas primitivas, se consideraba que la enferme- dad, al igual que la salud, era causada por la intervención de "espíritus", por lo que los ritos mágicos y sobrenaturales eran empleados para tratar de mantener la salud y curar la enfermedad.

Al estructurarse las diferentes sociedades y estados político-religiosos, la salud fue considerada como producto de la gracia divina y la enfermedad como un castigo divino. A pesar de ésta influencia, los griegos llegaron a comprender que la enfermedad era un proceso natural, fruto del desequilibrio en la naturaleza del individuo y que el organismo humano tenía la capacidad natural para restablecerlo. Aristóteles señala en su obra Metafísica que existe una salud objetiva y otra subjetiva; llegando a comprender a la salud y a la enfermedad como dos elementos contrarios de un mismo proceso, donde 
ambos estados eran producidos por una causa. Aunque Aristóteles no escribió acerca de la medicina de su tiempo, esta interpretación constituye el primer antecedente en la historia de las ideas donde se destaca la dinámica del Proceso Salud-Enfermedad.

Durante el oscurantismo de la Edad Media, e inclusive durante el Renacimiento, los conceptos de salud y enferme- dad continuaron con gran influencia mágico-religiosa, y es hasta el advenimiento de la ciencia aplicada a la tecnología que se llega a entender a la salud como la ausencia de enfermedad, y ésta, como un proceso causado por un agente biológico (etapa unicausal de la medicina). Estos conceptos prevalecieron por mucho tiempo en la ciencia médica; sin embargo, desde el siglo VII, la medicina proclamaba que los problemas de salud eran fenómenos sociales que en su desarrollo incluían tanto al individuo como a la sociedad. Esta corriente de pensamiento en la medicina fue ahogada por condiciones propias de la época, pero revivieron con el deterioro de la calidad de vida de la población y el surgimiento de la industrialización y de la clase obrera organizada.

Ya integrada la Organización Mundial de la Salud, se consideró al individuo como un ser integral, comprendiéndose la importancia de sus esferas biológica, social y psicológica para el mantenimiento de la salud, entendiéndose ésta como el completo bienestar físico, mental y social, y no sólo la ausencia de enfermedad.

Con la aparición de las nuevas ciencias que estudian al ser humano en relación con su medio ambiente, surge el concepto ecologista de la salud. Este pone de manifiesto el mecanismo de adaptación del individuo con base en un estado de tolerancia y compensación fundamentado en la interacción de la triada ecológica. Este modelo establece relaciones de multicausalidad basadas en el binomio susceptibilidad-resistencia del huésped, contribuyendo al origen del concepto de la Historia Natural y Social del Proceso Salud - Enfermedad.

Finalmente, dentro del esquema evolutivo descrito hasta aquí, mencionaremos por último que la salud se esta interpretando actualmente como un bien económico, mismo que da respuesta al paradigma económico que está dominando la interpretación del Proceso salud-Enfermedad.

Como vestigios de este desarrollo, en la actualidad han prevalecido diversos elementos conceptuales que permiten interpretar a la salud como:

a) Un estado que manifiesta una unidad biopsicosocial.

b) Un proceso de adaptación.

c) Un fenómeno sociocultural.

d) Un proceso que se observa dentro de un marco histórico determinado.

e) Un estado que refleja un alto contenido ideológico. 
El Proceso Salud-Enfermedad, producto del desarrollo histórico de la humanidad, pone a la ciencia médica en general, y al médico en particular, en el umbral de una práctica médica contradictoria, determinada básicamente por las relaciones de producción de la sociedad actual, salpicada de asistencia social y mistificada dentro de las bases del libre mercado.

Como se ha indicado brevemente, el concepto de salud y enfermedad han evolucionado constantemente a través de la historia, desde ser entendidos como una acción divina de gracia o castigo, hasta el concepto integral de la Organización Mundial de la Salud, que define al hombre como una unidad bio-psico-social. Esta evolución manifiesta que ambos conceptos son nociones relativas, adaptados a los criterios objetivos o subjetivos, tanto sociopolíticos como económicos y culturales para hacer frente a los problemas de salud y enfermedad. En el transcurso de este siglo, los conceptos de salud y enfermedad han dado un paso más allá de la capacidad de adaptación humana, analizándose como la manifestación de un fenómeno social, con un matiz político, histórico, ideológico, económico y cultural, por lo que la salud ha sido considerada no únicamente como un proceso de adaptación, sino también como un fenómeno social, individual y colectivo, producto de diversos factores, con implicaciones políticas, socioculturales, y económicas, dentro de un marco histórico, pero no podemos ignorar el componente ideológico del proceso de salud. Entonces la salud sería el producto de un proceso humano de equilibrio con la triada ecológica, dentro de un marco histórico específico, que permite al individuo realizar adecuadamente sus actividades de la vida diaria.

La enfermedad se vincula estrechamente con la salud, ya que representa el punto opuesto de ésta, permitiendo que sea graduada en la escala del Proceso Salud-Enfermedad. Debido a su papel como contrario a la salud en la dinámica de este proceso, la enfermedad puede ser analizada mediante los mismos elementos de la salud, y entenderla como el producto de un proceso humano desfavorable de adaptación con la tríada ecológica, dentro de un marco histórico, que impide al individuo la realización de sus actividades de la vida diaria.

La conceptualización del Proceso Salud-Enfermedad como un hecho social y dentro de un marco ideológico, conduce a ejercer la medicina como un instrumento de justicia social y a evaluar esta actividad profesional como un efecto del desarrollo social, pues la salud es un derecho humano fundamental y un objetivo social prioritario, además de que el Proceso Salud-Enfermedad es un fenómeno individual y colectivo, influenciado por factores y componentes biológicos, sociales e ideológicos, regulados por un proceso de adaptación. 
Así es como ha surgido una práctica médica dicotómica; por un lado la asistencia social institucionalizada, y por otro, la práctica médica privada, creyendo que la primera es la síntesis dialéctica de la segunda, sin entender que no es más que su propia contradicción histórica, por encontrarse ambas al servicio de un sistema de producción determinado, y traduciéndose en una desvinculación de la esencia de la medicina a nivel teórico y práctico, al mismo tiempo que el médico ha perdido su función social objetiva.

Sin embargo, la enfermedad continúa entendiéndose como un proceso de inadaptación o desequilibrio biológico- eco lógico, como resultado de la inexistencia, insuficiencia o ineficiencia de mecanismos de adaptación intrínsecos al ser humano como individuo o como grupo. Pero la enfermedad, al igual que la salud, no es únicamente un fenómeno biológico o ecológico, sino también un fenómeno social que debe ser interpretado dentro de un marco histórico específico, inmerso en un contexto sociocultural, político, económico e ideológico.

Aún en nuestros días, prevalece el conocimiento de que la salud y la enfermedad, son entidades independientes, donde la enfermedad se posesiona del individuo "como algo que 'se pesca' o que se 'pega"; una entidad que tiene existencia por sí misma. Esta idea es fomentada por las sociedades industrial izadas (a través de la desinformación), disminuyendo la demanda de servicios preventivos de salud, por lo que el perfil de enfermedades es un reflejo de la estructura social en que se desenvuelve la comunidad (dígase patología de la pobreza, de la riqueza, etc.); además de contribuir al sostenimiento del conocimiento 'legítimo' de las entidades nosológicas, como la materialización de un mito políticamente conveniente.

La salud y la enfermedad son momentos diferentes y opuestos de un mismo proceso; se excluyen uno a otro sutilmente y dentro de la dinámica social establecen relaciones dialécticas entre ambos. La práctica médica basada en este marco conceptual, permite comprender que la salud individual refleja en la salud colectiva, el ambiente (natural y social) que imprime sus efectos en la salud individual, desarrollándose relaciones recíprocas que generan un estado teórico de equilibrio. Al perderse éste, se manifiestan diversos grados de salud o enfermedad.

Sin embargo, la salud se ha interpretado como un mito políticamente conveniente, donde la enfermedad expresa las demandas de la sociedad industrial, además de reflejar su organización social prevalente. En este caso, la salud ya no es un don otorgado por la divinidad, ni aún el producto de una "influencia" que termina por posesionarse del ser humano, sino una meta cada vez más distante a la que uno tiene derecho en virtud de la justicia social. 
Salud, sano, saludable... son finalmente, palabras cotidianas que califican (cualitativa o cuantitativamente) actividades humanas, individuales o colectivas, éticas, morales o políticas. Así, tenemos diversos indicadores de salud que permiten evaluar el "NIVEL DE SALUD" de una comunidad, aun cuando estos indicadores miden la enfermedad y no la salud. Estos indicadores nos permiten saber cuánto o de qué se enferma una población, la cobertura de los servicios de salud, el número de médicos y de camas de hospital por número de habitantes, pero no se hace hincapié en la población sin acceso a servicios de salud, ni la distribución de enfermedades por clase social, por actividad productiva, por región socioeconómica, o en la concentración urbana de servicios de salud, sean preventivos, curativos o de rehabilitación.

\section{B) Cultura.}

El análisis histórico de la evolución del concepto y práctica de la salud nos permite comprender que la salud está determinada por un patrón cultural, entendida como los hábitos, costumbres y modos de vida de una sociedad, por lo que el concepto sobre salud-enfermedad, el conocimiento de la salud y la enfermedad, y los modos y estilos de vida, permiten cualificar las actividades de la vida diaria, los patrones culturales, el estilo de vida e inclusive, la calidad de vida. Así, nos encontramos entonces con un conjunto de hechos, un conjunto complejo de creencias, costumbres, estipulaciones e instituciones que pueden influir sobre el desarrollo del Proceso Salud-Enfermedad.

Así, la cultura no está ni simplemente yuxtapuesta ni simplemente superpuesta a la vida, en un sentido la sustituye, en otro la utiliza y la transforma para realizar una síntesis de un nuevo orden, por lo que hablar de cultura es hablar de un proyecto de vida, pues la cultura es todo lo que el hombre ha producido o modificado mediante el uso de sus facultades y de su esfuerzo, es decir, por medio de su actividad, lenguaje, costumbres, ideas, creencias y organización social.

El materialismo cultural, basado en la premisa de que la vida social es una reacción frente a los problemas prácticos de la vida terrenal, se constituye en una estrategia que permite comprender las causas de las diferencias y semejan- zas entre sociedades y culturas en el ámbito del Proceso SaludEnfermedad, por lo que la acción social, ya sea como una acción racional con arreglo a fines, una acción con arreglo a valores, una acción afectiva o una acción tradicional, contribuyen a valorar la participación del individuo y de la comunidad en el proceso de identificación de sus necesidades en salud y en la organización de auto cuidado que realiza el individuo o la comunidad para conservar, mejorar o restablecer el estado de salud. 
A raíz de la industrialización, se produjeron diversos cambios en las condiciones de vida y de trabajo, los medios de transporte, la atmósfera, la agricultura y hasta la preparación de los alimentos. El ser humano ha adoptado nuevas costumbres que no son saludables tales como la vida sedentaria, el tabaquismo, el consumo de alcohol y el uso indebido de drogas, sin contar con la sobrealimentación.

- Desde este contexto destaca en la población:

- El concepto que prevalece sobre la salud y la enfermedad,

- El conocimiento de la enfermedad,

- Las actitudes para conservar el estado de salud,

- Los estilos de vida,

- El padecer médico,

- La vivencia de la enfermedad,

- La percepción del acto de morir, que cuestiona el dónde y como se nace, y el dónde y cómo se muere.

- La percepción de la propia gravedad o no de la enfermedad, donde se experimenta la dualidad del afecto y la comunicación, es decir, lo que se dice es diferente a lo que se piensa.

- La actitud de protección hacia los extremos de la vida.

\section{El Problema:}

En resumen, un análisis histórico de la evolución de la atención a la salud permite concluir que ésta ha estado determinada por el patrón cultural prevalente, por lo que se percibe que ambas variables (cultura y salud) establecen una interrelación bidireccional que permite condicionar o determinarse una a la otra.

Dentro de este contexto, surge la necesidad de analizar las relaciones que establecen la cultura y la salud, para propiciar la aparición de una respuesta social organizada que enfrente la problemática de salud que manifiesta la sociedad.

\section{El Paradigma de la transición}

Analizar las relaciones que se establecen entre la cultura y la salud implica meditar tanto del lado del enfermo como del sano, ya sea para identificar las posibles vías para su curación, para conservar o mejorar su estado de salud; de aquí se sigue que si queremos identificar las relaciones que establecen ambas variables debemos meditar, reflexionar, acerca de ellas y propiciar la transfor- 
mación de los modelos previamente establecidos, lo que es en sí, un proceso difícil y complejo, y algunas veces doloroso.

La salud es un mirador desde el cual se pueden observar muchos de los procesos sociales, económicos y políticos de nuestro tiempo, representando un punto de confluencia donde se articulan la política económica y social, el individuo y la comunidad, lo público y lo privado, la eficiencia y la equidad, el conocimiento y la acción.

El amplio campo del Proceso Salud-Enfermedad, así como el de la cultura, puede ser observado desde el sistema de salud, por lo que debemos recordar que entre los principales logros del Sistema Nacional de Salud puede destacarse el combate a la enfermedad, la cobertura de los servicios y el desarrollo institucional, sin embargo, se espera que en lo cultural la diseminación de las explicaciones racionales y la constatación del poder de la tecnología produzcan mayores expectativas sobre la calidad de vida y mayor aceptación de las intervenciones científicas sobre la enfermedad. Sin embargo, esta interpretación se centra dentro de un vínculo profundo entre salud y economía, donde se interpreta a los servicios de salud con una naturaleza dual, constituyendo por una parte un componente fundamental del desarrollo y el bienestar social, y por otra, forman un creciente sector de la economía.

Todo sistema o subsistema social posee características que se reflejan (individual y colectivamente) en las condiciones de salud y enfermedad, en los riesgos de vida y en la calidad de la misma. Para interpretar la interacción de todos estos eventos, es necesario reconceptualizar el marco teórico que fundamente el análisis de sus indicadores entendiendo la dinámica del Proceso Salud-Enfermedad, dentro de la naturaleza biológica y social en la que se desenvuelve el individuo y su colectividad.

Debido al periodo de transición que transcurre actual- mente en México, se propone el paradigma de la "transición" que incluye los siguientes procesos de transición:

- Transición demográfica.

- Transición epidemiológica.

- Transición de la atención a la salud.

\section{a) Transición demográfica.}

La salud establece relaciones fundamentales entre el me- dio ambiente, el aumento de la población y las diversas modalidades de asentamiento s, por lo que al sufrir cambios la estructura sociodemográfica de la población, se observarán efectos considerables que repercutirán sobre la salud, por lo que 
los problemas principales con que se enfrenta la humanidad en los lugares en que vive están estrechamente relacionados entre sí: la pobreza, el hambre, el crecimiento demográfico, la urbanización no planificada, el deterioro del medio ambiente, la enfermedad, el analfabetismo, entre otros. México está experimentando dos procesos de enorme trascendencia social: primero, un envejecimiento de la población mucho más rápido que el que tuvieron los países actualmente desarrollados; segundo, una acelerada y en ocasiones desordenada urbanización que genera riesgos para la salud al tiempo que aproxima a la población hacia los principales núcleos donde se concentran los recursos médicos.

\section{b) Transición epidemiológica.}

Desde el punto de vista social, lo ideal sería una vida larga y productiva que culminara en un súbito colapso terminal; una vitalidad que persiste hasta el final y una muerte que llega sin temor ni tragedia al término natural de la vida del sujeto. Aunque parece poco probable que este ideal se convierta en norma, lo que si sería posible es reducir la morbilidad en la última fase de la vida; orientando a comprender una vitalidad creciente y de una duración limitada de la vida con la consiguiente comprensión de la morbilidad en un breve período previo al final fatal.

Algunas enfermedades graves no transmisibles, como el cáncer de pulmón y las cardiopatías, son cada vez más frecuentes en los países en desarrollo. Ello se debe, entre otras causas, a que la población abandona sus formas de vida tradicionales para adoptar las de los países industrializados.

La transición epidemiológica implica el cambio de morbilidad en las siguientes direcciones:

- Una disminución de las carencias e infecciones nutricionales, como también de las tasas de mortalidad de niños y jóvenes.

- Un aumento de caries dental, obesidad, hipertensión, diabetes y enfermedades vasculares.

- Un aumento de las enfermedades gastrointestinales, trastornos malignos del intestino grueso, diverticulítis y apendicitis.

- Una disminución de la tasa de ciertos tipos de cáncer, compensada con creces por un aumento de muchos otros.

Padecemos el traslape de dos retos: de un lado, terminar con el rezago representado por las enfermedades del subdesarrollo, tales como las infecciones comunes, la desnutrición y las muertes maternas y perinatales; del otro, enfrentar los retos emergentes asociados a la industrialización y la urbani- 
zación, tales como las enfermedades cardiovasculares, el cáncer, los padecimientos mentales, las adicciones y las lesiones.

\section{c) Paradigma de modelos de atención médica para hablar sobre la transición de} la atención en salud

Aún cuando la enfermedad es un fenómeno inherente a la vida misma, sus manifestaciones son diferentes en las diversas formaciones históricas, económicas y sociales; por lo que los modelos de atención a la salud se han identificado como la realización pública de la asistencia médica, desarrollo que se ve obstaculizado por la ausencia de categorías conceptuales y adecuadas al estudio del Proceso Salud-Enfermedad, propiciando que las actividades intervencionistas del sector salud se conviertan en instrumentos ideológicos del Estado.

La transición en la atención a la salud, refleja el cambio de la demanda del tipo de servicios, y la satisfacción del paciente, debido a que algunos curadores tradicionales siguen funcionando como integrado res culturales, con una tendencia intervencionista, desacralizadora de la enfermedad, y comercializadora de las prácticas curativas aún de las tradicionales. Esto ha propiciado la construcción de una escala de prestigio entre los curadores, según la cual la medicina "moderna” representa el nivel más jerarquizado. Esta medicina expresaría los símbolos de la clase privilegiada, así como la de mayor valor de la ciudad y de su posición más alta.

Por otro lado, esto no ha limitado que la ampliación de los espacios de participación social exprese el reclamo de la atención a la salud como un derecho social y en la exigencia por servicios de alta calidad, incluyendo una mayor libertad para elegir al prestador.

Teniendo presente la dicotomía de la asistencia médica, se ha propuesto la integración de un Sistema Universal de Salud (SUNSalud), con la misión de mejorar la salud, reducir las desigualdades, controlar los costos y satisfacer a la población. El SUNSalud es en suma, la expresión de un "Pacto Social por la Salud", que permitiría instituir un mecanismo de convergencia para conducir la reforma del sistema de salud.

\section{Relaciones entre Cultura y Salud Vistas a Partir del "Paradigma Transicional”}

La relación entre cultura y niveles de salud es un asunto complejo, de múltiples dimensiones; sin embargo, la asociación entre actitudes culturales y la morbimortalidad es clara en el sentido de que ha mejores actitudes se obtienen mejores índices de salud. 
Aunque resulta relativamente fácil establecer la distinción de principio, la dificultad comienza cuando se quiere efectuar el análisis. Esta dificultad es doble: por una parte, se puede intentar definir, para cada actitud, un elemento de salud-enfermedad; por otra, buscar el mecanismo que per- mite que las actitudes culturales se inserten en comportamientos que son, en sí mismos, factores de riesgo dentro del Proceso Salud-Enfermedad.

Dentro de los límites siempre históricos y geográficos de la cultura (coexistencia en el tiempo y en el espacio con la especie biológica), y los fundamentos doctrinario s de la Fundación Mexicana para la Salud (FUNSALUD), las relaciones que se establecen entre la cultura y la salud pueden definirse como:

- Inclusión social: Se ha identificado que existen desigualdades importantes en cuanto a cobertura de salud en las diferentes entidades federativas, observando se una polarización de la cobertura de Atención Primaria de Salud. En una sociedad como la mexicana, de contrastes tan profundos, es indispensable que la atención a la salud represente una instancia donde todos reciban un trato semejante ante necesidades semejantes, partiendo de la posición ética del valor de la vida humana que permite generar una atención a la salud inclusiva, jugando un papel político y cultural de primer orden, fortaleciendo la identidad cultural, siendo regulado solamente por la necesidad de atención.

- Igualdad de oportunidades: la atención a la salud debe ayudar a que cada generación entre a la vida con las mismas oportunidades. En este sentido, asegurar a todos un piso básico de atención a la salud adquiere el mismo sentido de justicia que la educación. La igualdad de oportunidades ofrece la base ética para que la distribución de la riqueza sea socialmente justa.

- Autonomía individual y familiar: En la tipología de los riesgos a la salud existen diferencias a nivel individual, pues la conducta de los menos informados generalmente está sometida a presiones que fácilmente les inducen a deteriorar su salud. A esto se suman características socioeconómicas, las cuales pueden limitar de manera importante su participación en la búsqueda de un equilibrio entre las necesidades y su manejo intracomunitario. Por lo que toda persona tiene la libertad para decidir lo que más le conviene, prerrogativa que asume la familia en el caso de los menores y de las personas limitadas en su capacidad para decidir.

- Corresponsabilidad social: La participación en los grupos mejor informados es cualitativamente superior, más eficiente y justa que en los grupos que son dirigidos sin disponer de opciones y criterios sólidos. De ahí que se plantee como indispensable la socialización del conocimiento, también a diferentes niveles, incluyendo el comunitario y el de los recursos institucionales clave, para la orientación y guía de la participación comunitaria. Esta relación 
pone límites al anterior, pues toda libertad conlleva una responsabilidad. Ello es especialmente importante en el caso de aquellos bienes, como los servicios de salud, que producen lo que los economistas llaman "externalidades", esto es, consecuencias para los demás de las decisiones propias. Así, el hecho de que una persona no cuide su salud tiene efectos sobre los demás. Ello genera una responsabilidad que limita la libertad de no atenderse. Por otra parte, el carácter generalmente involuntario de las pérdidas de salud impone a los que están sanos la responsabilidad de cuidar de los enfermos.

- Cultura + salud = bienestar. La renovación de las ciencias y artes ha contribuido a la depuración de las costumbres. En el caso de México, los problemas de desigualdad social que ha originado el estilo de desarrollo económico se han reflejado en el nivel de salud de la población. El rezago de las condiciones de salud y el deterioro de los servicios con base en los referentes internacionales es un asunto que debe ser resuelto tras una clara decisión política. Esto nos induce a recordar que el Estado de Bienestar es un ausente, o su presencia circunstancial, fragmentada y/o acotada, a dado paso en América Latina a un "Estado de Malestar" pues se ha sufrido durante el decenio de los ochenta un severo revés en términos de un grave retroceso económico que ha acentuado el endeudamiento externo, elevando los procesos inflacionarios y agudizando las desigualdades sociales.

El "Estado de Malestar" posee una dimensión psicosocial generada en la conformación de una sociedad más dual en donde los estratos medios tienden a desvanecerse, y en donde se congelan las perspectivas de movilidad social ascendente. Sobre la comprobada ausencia de los servicios sociales básicos del estado en los sectores de menores ingresos, se construye un discurso de un individualismo dogmático que deja a cada persona liberada a su propia suerte, resistiendo el tejido social y las redes comunitarias de solidaridad. Esta dimensión conduce a una percepción colectiva o estado de ánimo que se traduce en un estado de descreimiento, que ambos contribuyen a una reducción de las expectativas relacionadas con el mejoramiento de la calidad de vida.

$\mathrm{Al}$ interior de las comunidades existen dinámicas que tienden a agravar o solucionar la problemática del Proceso Salud-Enfermedad que deben ser analizadas. Aquí resaltan las dimensiones de la comunidad, la familia y su organización para generar y distribuir recursos, que influencian a la salud y se manifiestan como redes locales y auto cuidado. 


\section{Respuesta social organizada para la salud}

La salud, como se ha visto, está determinada por la organización social. Una sociedad sana es la que desarrolla las capacidades del hombre; una sociedad insana es la que menosprecia la vida humana y convierte al hombre en instrumento de uso y de explotación para otros; por lo que para referimos a la respuesta social organizada para la salud, evocare el concepto de la Atención Primaria a la Salud, expresado en la Declaración de Alma Ata en 1978:

La atención primaria de la salud es la asistencia sanitaria esencial basada en métodos y tecnología prácticos, científicamente fundados y socialmente aceptables, puesta al alcance de todos los individuos y familias de la comunidad, mediante su plena participación y a un costo que la comunidad y el país puedan soportar en todas y cada una de las etapas de su desarrollo, con un espíritu de autorresponsabilidad y autodeterminación. La atención primaria, a la vez que constituye la función central del sistema nacional de salud, es el elemento central del desarrollo social y económico de la comunidad. Representa el primer nivel de contacto entre los individuos, la familia y la comunidad con el sistema nacional de salud, llevando lo más cerca posible la atención sanitaria al lugar en que residen y trabajan las personas, y constituye el primer elemento de un proceso permanente de asistencia sanitaria.

Todo lo señalado hasta aquí: lucha ideológica, diferentes objetos de estudio, distintas etapas, todo en presencia de una crisis que se instala con velocidad y que acarrea consigo la falta de recursos, convierte este terreno de necesidades en otro de especulaciones y confusión, que adolece de una política carente de objetivos claros. Esto es lo que ha acontecido con esta área en nuestro país.

Con el propósito de vincular estrechamente a la salud con el desarrollo económico y social, el "paradigma transicional” permite identificar la necesidad de promover la adopción de estilos saludables de desarrollo, permitiendo el pluralismo en la atención a la salud, mediante la libre elección del prestador de servicios de salud, fomentando el establecimiento de "Organizaciones para la Protección de la salud”, que compitan por la prestación de servicios a grupos de personas dentro de un ambiente eminentemente social, donde la promoción de la salud mediante la prevención de riesgos conlleva el análisis y priorización de los estilos de vida como un elemento fundamental del auto cuidado de la salud.

Se retorna a la familia y a distintos organismos de la sociedad civil, sin fines de lucro, como efectores de salud; sin embargo, esto se puede llegar a realizar desde el desmantelamiento y la renuncia explícita del Estado a asu- 
mir su responsabilidad en términos de una provisión de servicios de salud y educación sobre bases universales; por lo que se favorecen sistemas de auto ayuda no por sus meritos organizativos-participativos, sino por su nula significación financiera para el sector público. Falta una transferencia de conocimientos y prácticas a las familias y comunidades, de modo que ellas puedan enfrentarse a los problemas de salud y educación que las aquejan, por lo que la participación comunitaria, la descentralización y la privatización total o parcial de los servicios de atención a la salud no representan más que una transferencia de problemas.

La profesión médica es una manifestación, en un sector particular, del control sobre la estructura del poder de clase que han adquirido las élites universitarias; por lo que la respuesta social organizada debe contemplar la equidad, la utilización y el acceso a los satisfactores básicos relacionados con la salud, que al articularse, inserte a los determinantes de la salud vistos como elementos que participan en un proceso que genera permanentemente el conjunto de comportamientos sociales, entre los que se encuentra el origen y la manifestación de los riesgos de la salud.

\section{Conclusiones}

Conclusión 1: La interacción entre el individuo y su medio ambiente está enlazada y regulada por mecanismos de adaptación que permiten al individuo, y al grupo que pertenece, responder favorable o desfavorablemente a los estímulos que provienen del ambiente (eco sistema o antroposistema), con el fin de mantener un equilibrio entre las distintas esferas del individuo, el macro y el microambiente, dentro de los límites normales.

Conclusión 2: El estado de salud implica una cultura en salud que no excluye la individualización, sino justamente la exige, con el propósito de que en materia de atención a la salud deje de existir el racionamiento.

Conclusión 3: El conservar, mejorar o restablecer el estado de salud puede llegar a ser una espiral de superaciones personales y comunitarias constantes; donde el proceso de auto cuidado se realiza siempre en mudanza rítmica de movimientos contrapuestos, que pueden ser calificados como de inspiración y expiración, de acción y reacción, de padecer y hacer, de dar y tomar, de gozar y renunciar.

Conclusión 4: Si aceptamos la existencia del "paradigma transicional" como se ha definido aquí, se permite destacar el papel que el concepto de salud-enfermedad, el estilo de vida, los conocimientos de salud y enfermedad, contribuyen a regular una atención a la salud sobre un criterio de prioridad 
socialmente percibida, comprometiendo a la sociedad en general a promover el auto cuidado de la salud como un esquema cultural predominante que incluye la interacción de áreas como la alimentación, la educación, el empleo, la vivienda, la recreación, el medio ambiente y la participación del cuidado de la salud de toda la comunidad.

Conclusión 5: La "respuesta social organizada para la salud", no puede apartarse del contexto cultural que propicia el propio desarrollo, orientándose a interpretar esta relación fundamentalmente como una relación donde la cultura y la salud se sinergizan para dar paso a un bienestar social, fundamentado en la conservación o mejoramiento de la calidad de vida del individuo.

Este análisis permite concluir finalmente que la "respuesta social organizada" es un elemento esencial para proporcionar una atención médica que permita la satisfacción de los diversos grupos segregados y diferenciados desde la perspectiva de su propia cultura en salud, pero además es importante expresar la necesidad de estudiar más las medidas de gratificación del comportamiento saludable, es decir, de la "desmedicalización". Aunque resulten conflictivas, esas medidas pueden disminuir la demanda de atención de salud oficial y reducir el problema financiero mientras no se integren al sistema oficial las nuevas alternativas de atención de salud. Para prevenir hay que comprender las reacciones humanas: nuestra relación con los inconvenientes de la vida (nacer, crecer, envejecer y morir) y con las diversas enfermedades (desde el resfriado común hasta un cáncer en fase terminal); por lo que tal vez la solución sea reconocer que los valores culturales son uno de los instrumentos para alcanzar la salud para todos, respetando el ámbito de la libertad.

Finalmente, debemos preguntamos cómo el comportamiento de la población, sus conocimientos, sus actitudes y sus costumbres influyen en su estado de salud, recordando que este es un componente de la calidad de vida entendida como las condiciones de vida que resultan de la combinación de los efectos de toda una serie de factores determinantes de la salud, la felicidad (que incluye un medio físico agradable y una ocupación satisfactoria), la educación, las realizaciones sociales e intelectuales, la libertad de acción, la justicia y la ausencia de opresión; por lo que la salud debe considerarse como una materia multidisciplinaria o transdiciplinaria y no puede ser prerrogativa de una profesión con exclusión de las demás.

Recordando a Pestalozzi: "Probadlo todo, conservad lo bueno y si algo mejor ha madurado en vosotros mismos, añadidlo en verdad y amor a lo que yo intento daros..., en afanes de mi vida como un objeto que, ya terminado, no necesita más prueba. Aun no está verdaderamente terminado y necesita con toda seguridad una prueba seria y ciertamente no por mi y por mis ruegos." 
Convergencia Revista de Ciencias Sociales, núm. 12/13, 1996, Universidad Autónoma del Estado de México

\section{Bibliografía}

Bakan, D., 1979: Enfermedad, Dolor, Sacrificio. Hacia una psicología del sufrimiento, México: Fondo de Cultura Económica.

Bustelo, E.S., 1991: "La producción del estado de malestar. Ajuste y política social en América Latina” en Salud Pública N³3, México.

Engel, G.L., 1977: The need for a new medical model A challenge for biomedicine, en Science $\mathrm{N}^{\circ} 19$.

Engel, G.L., 1982: The biopsychosocial model and medical education: New Ing J Med.

Frenk J, et. Al., 1991: “Elementos para una teoría de la transición en salud” en Salud Pública $\mathrm{N}^{\circ} 33$, México.

Frenk J, R Lozano, Ma. González-Block et al., 1994: Economia y Salud:propuestas para el avance del sistema de salud en México. Informe Final, México: Fundación Mexicana para la Salud.

Fries, J.J., 1985: La comprensión de la morbilidad en Foro Mundial de la Salud $\mathrm{N}^{\circ} 6$.

Gutiérrez, M., 1979: El Hombre. Introducción a la antropología filosófica. México: Editorial Font.

Hernández-Peña P, O. Zapata, R. Leyva y R. Lozano, 1991: "Equidad y salud: necesidades de investigación para la formulación de una política social" en Salud Pública $\mathrm{N}^{\circ} 33$.

Ilich, 1., 1978: Némesis Médica. La expropiación de la salud, México: Joaquín Mortíz.

Jamison, D.T., and W.H. Mosley, 1991: Disease control priorities in developing countries: health policy responses to epidemiological change, en Am $\mathrm{J}$ Public Health $\mathrm{N}^{\circ} 81$.

Kuhn, T.S., 1975: La Estructura de las Revoluciones Cientificas, México: Fondo de Cultura Económica.

Levi-Strauss, C., 1985: Las Estructuras Elementales del Parentesco, México: Editorial Artemisa.

Löbsack, T., 1986: Medicina Mágica. Métodos y méritos de los curanderos milagrosos, México: Fondo de Cultura Económica.

Loewe R., 1988: "Atención Primaria a la Salud: revisión conceptual” en Salud Pública $\mathrm{N}^{\circ}$ 30, Méx.

Menéndez, E: L., 1981: Poder, Estratificación y Salud. México: Ediciones de la Casa Chata.

Molina Salazar, R.E., J.R. Romero Velázquez y J.A. Trejo Rodríguez., 1991: Desarrollo Económico y Salud en Salud Pública N³3, México. 
Ortiz Quezada, F., 1986: La Medicina y el Hombre, México: Universidad Autónoma Metropolitana.

Ortiz Quezada, F., 1989: El Acto de Morir. Sobre la muerte de Ivan Ilich de León Tolstoi, México: Némesis.

Osuntokun, B.O., 1985: "El Cambio de los Tipos de Morbilidad en los Países en Desarrollo" en Foro Mundial de la Salud N 6.

Ramalingaswami, V., 1984: "La Salud Sin la Prosperidad" en Foro Mundial de la Salud $\mathrm{N}^{\circ} 5$.

Ramírez Torres, J.L., 1995 Codificación y Códigos Culturales. Simbólica y ritual en cultos curativos. Tesis Doctoral, México: Facultad de Filosofía y Letras e Instituto de Investigaciones Antropológicas, Universidad Nacional Autónoma de México.

Rillo, A.G., 1992: "Miseria de la Medicina” en Humanidades No. 30, Toluca: UAEM.

Rillo, A.G., 1992: "El Proceso Salud-Enfermedad, un Fenómeno Social” en Humanidades No. 48, Toluca: UAEM.

Rillo, A.G., 1992: "La Salud Más Allá de la Adaptación" en Humanidades No. 50, Toluca: UAEM.

Rillo, A.G., 1992: "Indicadores del Proceso Salud-Enfermedad y las Condiciones Materiales de Vida" en Revista de la Facultad de Medicina $\mathrm{N}^{\circ} 3$ Toluca, UAEM.

Rillo, AG., 1993: Salud y Enfermedad: reflejo de la estructura social. En Humanidades No. 52, Toluca: UAEM.

Rillo, A.G., 1996: "Análisis teórico del informe final de FUNSALUD: Economía y Salud” en La Mora N ${ }^{\circ}$ 20, Toluca, UAEM.

Rillo, A.G., E. Ortíz-Romo y J. Sánchez, 1991: "Guerrero: Morbilidad en Instituciones Penitenciarias. En LXXII Reunión Reglamentaria de la Asociación de Investigación Pediátrica.

Rillo, A.G., E. Ortíz-Romo y J. Sánchez-Guerrero, 1991: “Influencia de las Condiciones Materiales de Vida en Instituciones Penitenciarias" en $\mathrm{Me}$ morias del Congreso Bienal de la Federación Mundial en Salud Mental, México, FMSM.

Rillo A.G., E. Ortíz-Romo, J Sánchez-Guerrero y L.C. Bermúdez, 1992: "Perfil Epidemiológico de Instituciones Penitenciarias" en Revista del Residente de Psiquiatría $\mathrm{N}^{\circ} 2$.

Sagaon, V.F. y Rillo A.G., 1990: "Valoración del peso intrauterino en recién

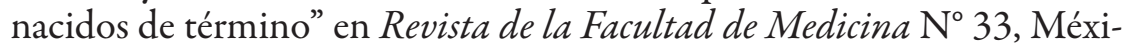
co: UNAM. 
Solis-Márquez, M.E. y A.G. Rillo, 1990: "Morbilidad en instituciones penitenciarias" en Revista de la Facultad de Medicina $\mathrm{N}^{\circ} 33$, México: UNAM Spranger, E., 1966: Cultura y Educción, España: Espasa Calpe.

Valencia, J.M, M. V. José, J. Olvera, O. Serrano y A. Márquez, 1991: “Regionalización de México basada en indicadores de atención primaria a la salud" en Salud Pública N$^{\circ} 33$.

Vandale Toney, S., J.L. Duran Arenas y H. Ortega Minor, 1985: “La medición del estado de salud de la población: una actividad fundamental para los servicios de salud" en Salud Pública N 27. 